\title{
U.S. Naval Observatory Double Star CD 2006.5
}

\author{
Brian D. Mason and William I. Hartkopf \\ Astrometry Department, U.S. Naval Observatory, 3450 Massachusetts Avenue, NW, \\ Washington, DC 20392-5420, USA \\ email: (bdm,wih)@usno.navy.mil
}

\begin{abstract}
The U.S. Naval Observatory has produced its second CDROM of double star catalogs. This successor to the 2001.0 CDROM includes the latest versions (June 30 2006) of four major double star catalogs maintained at the USNO:

- Washington Double Star Catalog (WDS),

- Second Photometric Magnitude Difference Catalog,

- Fourth Catalog of Interferometric Measurements of Binary Stars, and

- Sixth Catalog of Orbits of Visual Binary Stars.

Each of these catalogs had seen significant changes during the past six years; for example, the WDS has grown by over 150,000 measures and the number of systems in the Interferometric Catalog has nearly doubled. Other improvements include precise coordinates for the vast majority of systems, as well as new observing lists for tens of thousands of "neglected" doubles.

Also included on this CDROM is a Catalog of Linear Elements for several hundred optical pairs. These elements should prove useful for improving the components' proper motions, as well as providing scale calibration out to several tens of arcseconds.

As was done with its predecessor, the new CDROM is automatically distributed free of charge to members of the double star community and to astronomy libraries. Others may receive a complementary copy upon request.
\end{abstract}

Keywords. binaries (including multiple): general, binaries: visual, catalogs, history and philosophy of astronomy

\section{Washington Double Star Catalog, 2006.5}

The WDS summary catalog is a listing of 102,387 systems based on 727,726 mean positions. In addition to the summary data we also provide precise positions and proper motions, as well as cross reference identifications. We also provide more detailed notes as well as indicating when systems are also in other datasets. The WDS is the successor to Index Catalog of Double Stars (IDS; Jeffers, van den Bos \& Greeby 1963) and has gone through three major releases (Worley \& Douglass 1984, 1996; Mason et al. 2001). It is under continual growth and is updated nightly. Statistics for these catalogs are presented in Figure 1.

In recent years the WDS has been matched with astrometric catalogs, primarily those used for the determination of proper motion for Tycho-2. These catalogs have significantly improved the identification of pairs with their precise positions, such that $97 \%$ of the systems in the WDS now have at least arcsecond-precise positions. Also, the proper motion of the secondary is now provided for $38 \%$ of all pairs, aiding in the identification of common proper motion binaries and optical doubles. Pairs having common or mutually exclusive parallaxes are also indicated.

In addition to the Durchmusterung cross references in the summary catalog, the WDS 


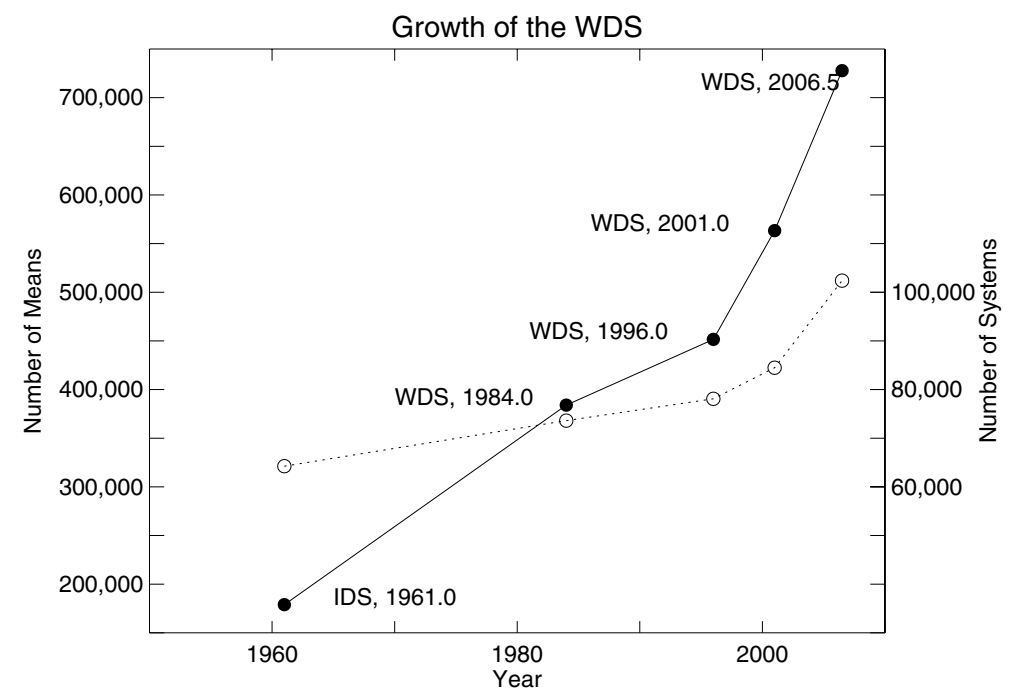

Figure 1. Growth of the WDS in terms of total measures (solid line) and systems (dotted line) at the time of each major release.

also provides cross references to Hipparcos, Tycho-2, and historical double star catalogs (ADS; Aitken (1932) \& BDS; Burnham 1906) via ancillary files.

New lists of "Neglected Doubles" have been created. Pairs are classified as neglected if they are unconfirmed or infrequently observed. Observation of these pairs by amateurs has significantly improved the database; yhtough their searched for these systems, amateurs have also provided arcsecond-precise coordinates for many pairs too faint or with too poor initial coordinates to match against astrometric catalogs. Unpublished measures of doubles from various sources are included on the CD, as well as lists of verified single stars.

Although the mean number of measures per system in the WDS database is 7.1 , the median is only 3. The distribution of these is presented in Figure 2. Some 1,522 pairs have orbits of varying quality, 354 have common parallax, and an unknown number have common proper motion. Many others are certainly optical: 1163 have rectilinear solutions and 174 have mutually exclusive parallax. With only a few percent even approximately described kinematically, there still remains much to be done.

\section{Sixth Catalog of Orbits of Visual Binary Stars}

The Sixth Catalog of Orbits of Visual Binary Stars continues the series of compilations of visual binary star orbits published by William Finsen $(1934,1938)$, Charles Worley (1963), Finsen \& Worley (1970), and Worley \& Heintz (1983) from the 1930's to the 1980 's. As of 30 June 2006 the new catalog included 2,024 orbits of 1,888 systems. All orbits have been graded as in earlier catalogs, although the grading scheme was modified as of the Fifth Catalog (Hartkopf, Mason, \& Worley 2001) to be more objective.

Nominally a single "best" orbit is given for each system; however, a second solution is occasionally given in cases of two very different orbits of similar quality or instances of quadrant ambiguity. Ephemerides are given for all orbits with complete elements, as are plots including all associated data in the WDS database. Examples of these plots are shown in Figure 3. Notes are given for many systems, and a subset of orbits potentially useful for scale calibration is also presented. 


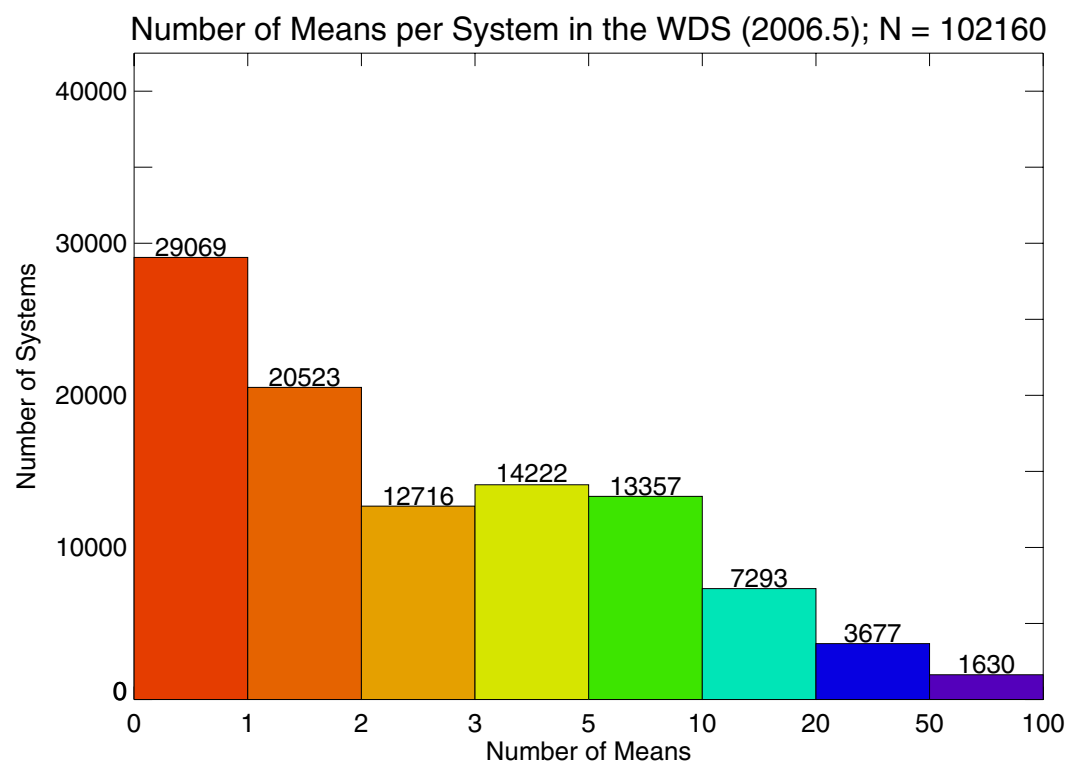

Figure 2. Histogram presenting the number of systems with the specified number of relative position determinations.

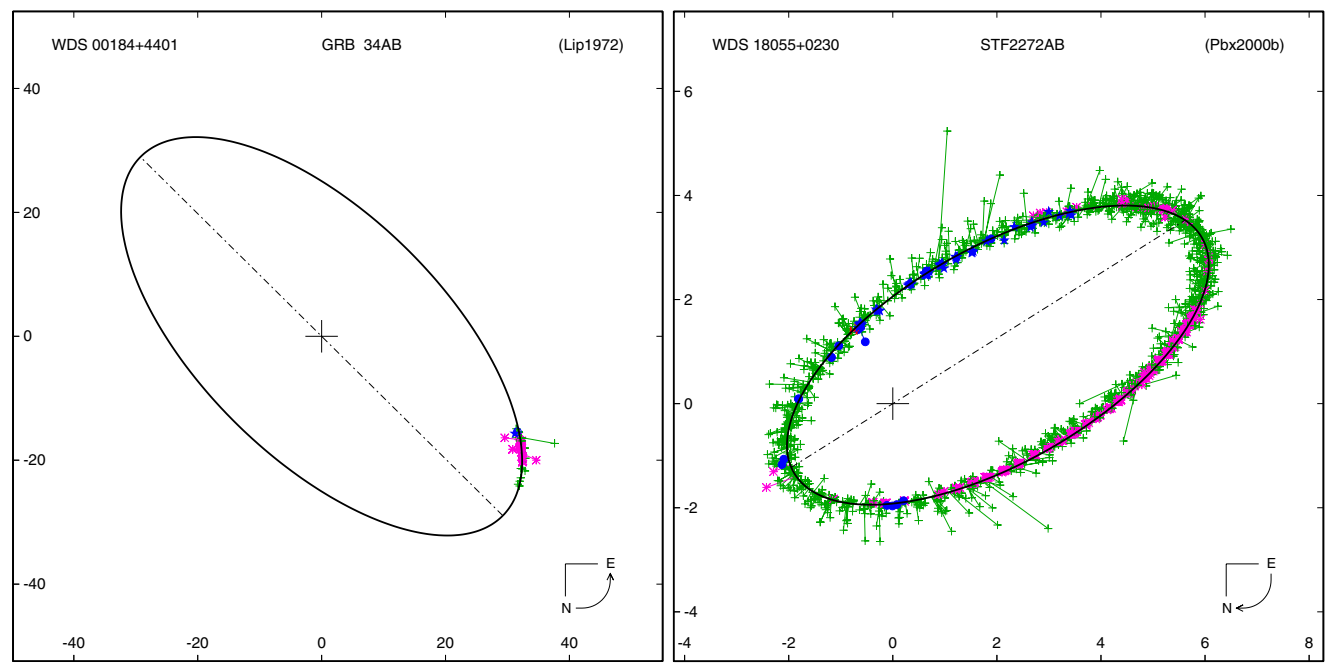

Figure 3. Left: The "Grade 5" (indeterminate) orbit for GRB 34. While residuals are small, and the ephemeris quite accurate for many years to come, the orbital coverage is slight and calculated astrophysical parameters would have large errors. Right: The "Grade 1" (definitive) orbit for STF 2272AB. Small residuals and excellent orbital coverage makes this among the best.

\section{Fourth Catalog of Interferometric Measurements of Binary Stars}

The Fourth Catalog of Interferometric Measurements of Binary Stars includes 104,618 published measures of binary and multiple star systems obtained by high-resolution techniques (speckle interferometry, photoelectric occultation timings, etc.), as well as 30,956 negative examinations for duplicity. Distributions of measures with time and separation are displayed in Figure 4. 

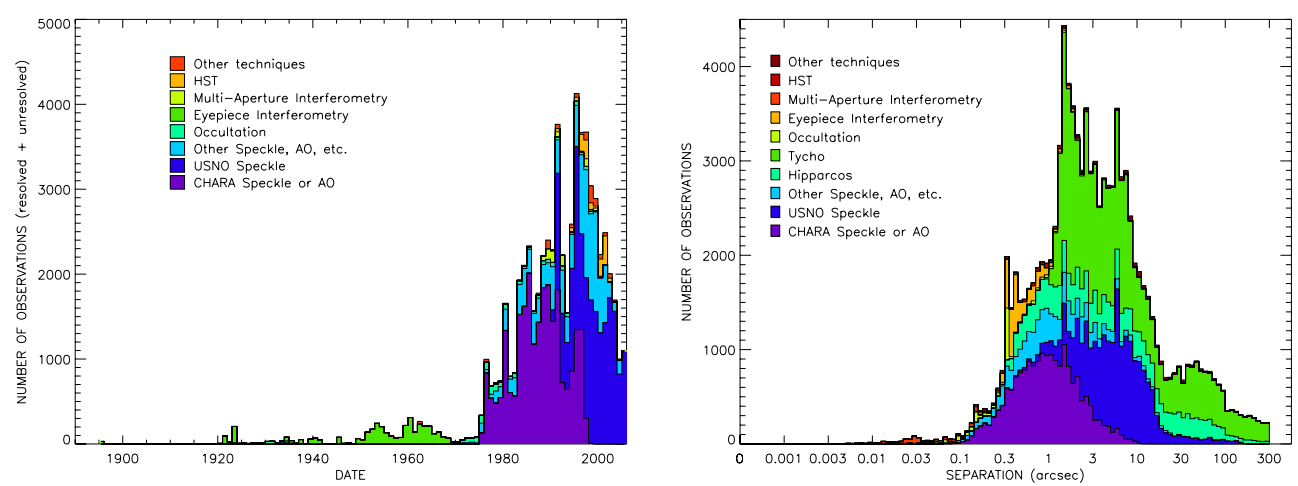

Figure 4. Left: Distribution of measures with time. Barely visible are the 1895 observations of Schwarzschild \& Villiger, followed two decades later by the 1919-1921 data obtained by Anderson and Merrill using the famous 20-foot beam interferometer with the Mount Wilson 100-inch Hooker Telescope. Right: Distribution of measures with separation, from the closest measures by long-baseline interferometry to the widest Tycho pairs.

\section{Second Photometric Magnitude Difference Catalog}

The Second Photometric Magnitude Difference Catalog is a collection of magnitude difference measures for double stars and serves as a repository for double star observations where no astrometry is given. It is ten times larger than the First Catalog (Worley, Mason, \& Wycoff 2001) and consists of 209,365 measures of 63,643 systems, with a mean $\Delta \mathrm{m}$ of 1.49. The most significant addition since the last version of this catalog resulted from matching the WDS against the 2MASS catalog. Delta-m determinations in $J_{-}, H$-, and $K$-bands are now listed for the 42,009 systems matched with WDS pairs. A brief summary and statistical analysis of the contents of the catalog are presented.

New on the Second Double Star CD are the following:

\section{Catalog of Rectilinear Elements of Visual Double Stars}

Many systems in the Washington Double Star Catalog have shown significant relative motion since their discovery. The Catalog of Rectilinear Elements provides linear fits for those systems whose motion does not appear to be Keplerian. While a few of these may in fact be very-long-period physical pairs whose orbital motion is not yet apparent, most are probably optical pairs (i.e., chance alignments of unrelated stars). These linear fits, then, just describe the relative proper motions between these pairs of stars. Examples of linear fits are given in Figure 5.

The purposes of this catalog are threefold. First, the very well-defined motions of some of these systems means they may prove useful for scale calibration for imaging systems such as CCDs or photographic cameras. Also, these differential proper motions may allow us to improve upon proper motions of individual components. Finally, these linear fits, especially in comparison with Hipparcos proper motions, may be useful in searches for submotions due to closer components. These investigations are underway. Fourier analysis of residuals to linear fits may also indicate the presence of unseen companions. 


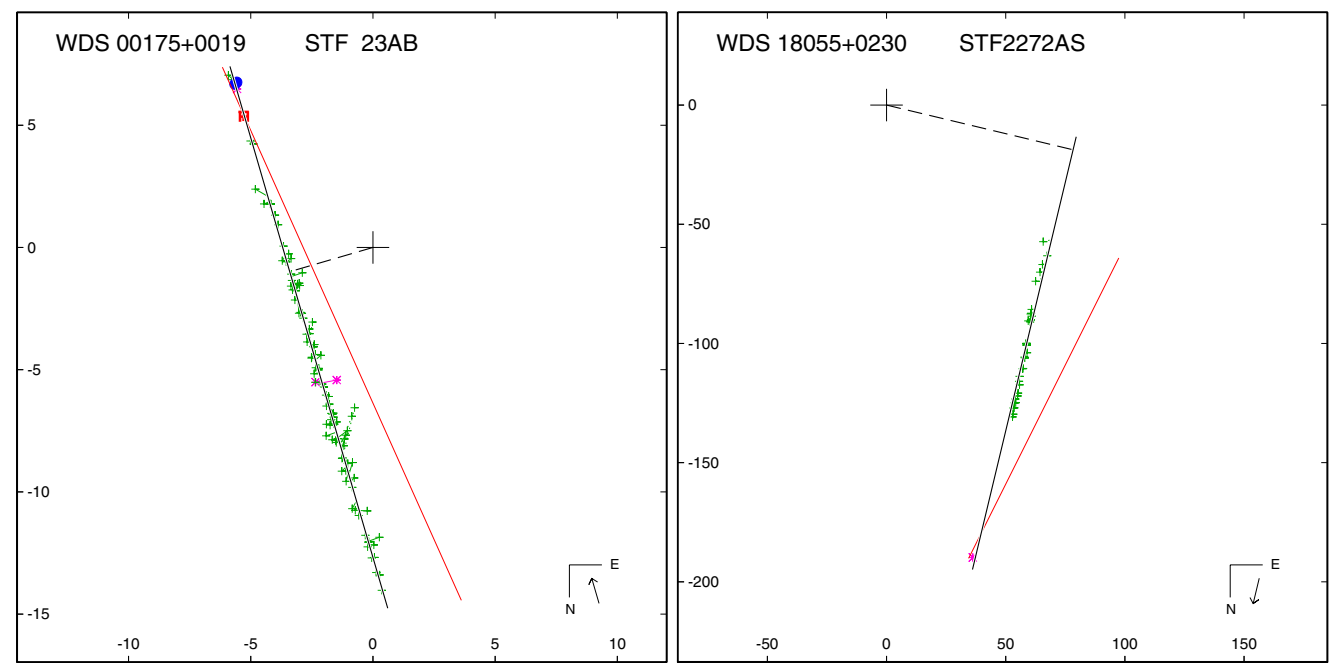

Figure 5. Left: STF 23, whose 170+ years' worth of data illustrate a clear deviation from the published relative proper motion (red line). For this class of objects, the long timebase of double star measures may yield better proper motions than traditional techniques. Right: STF 2272AS, a distant pair exhibiting a "wobble" in its motion due to the orbit of a closer pair (seen in the orbit in Figure 3).
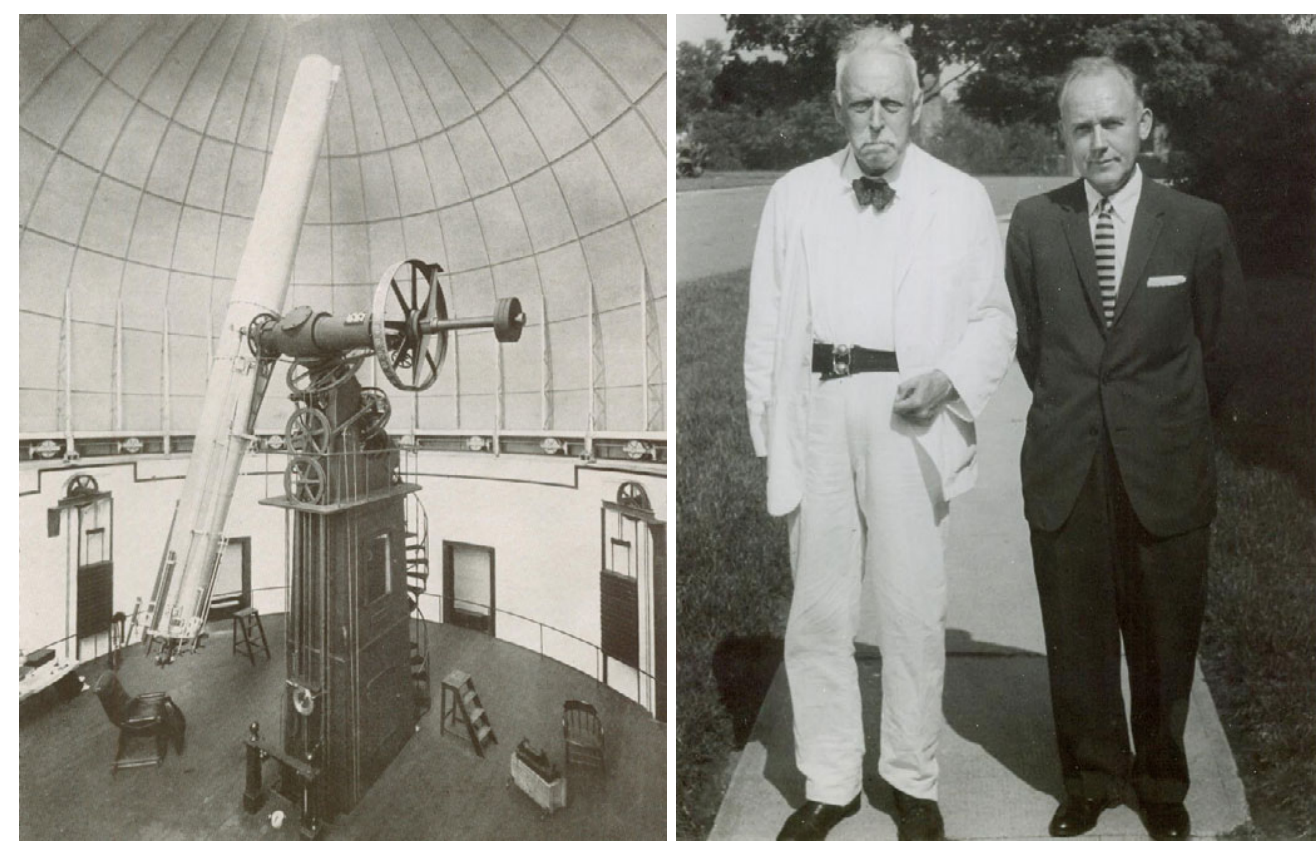

Figure 6. Left: The $26^{\prime \prime}$ in 1911. Right: Ejnar Hertzsprung visiting the USNO, seen with Scientific Director, Kaj Strand.

\section{Double Star Astronomy at the US Naval Observatory}

The U.S. Naval Observatory has, for well over a hundred years, been involved in various programs related to the observation of double stars. Highlights of these efforts, using the three observing techniques of visual filar micrometry, photography, and speckle interferometry, are given, along with many historic photographs (see Figures 6 and 7). 

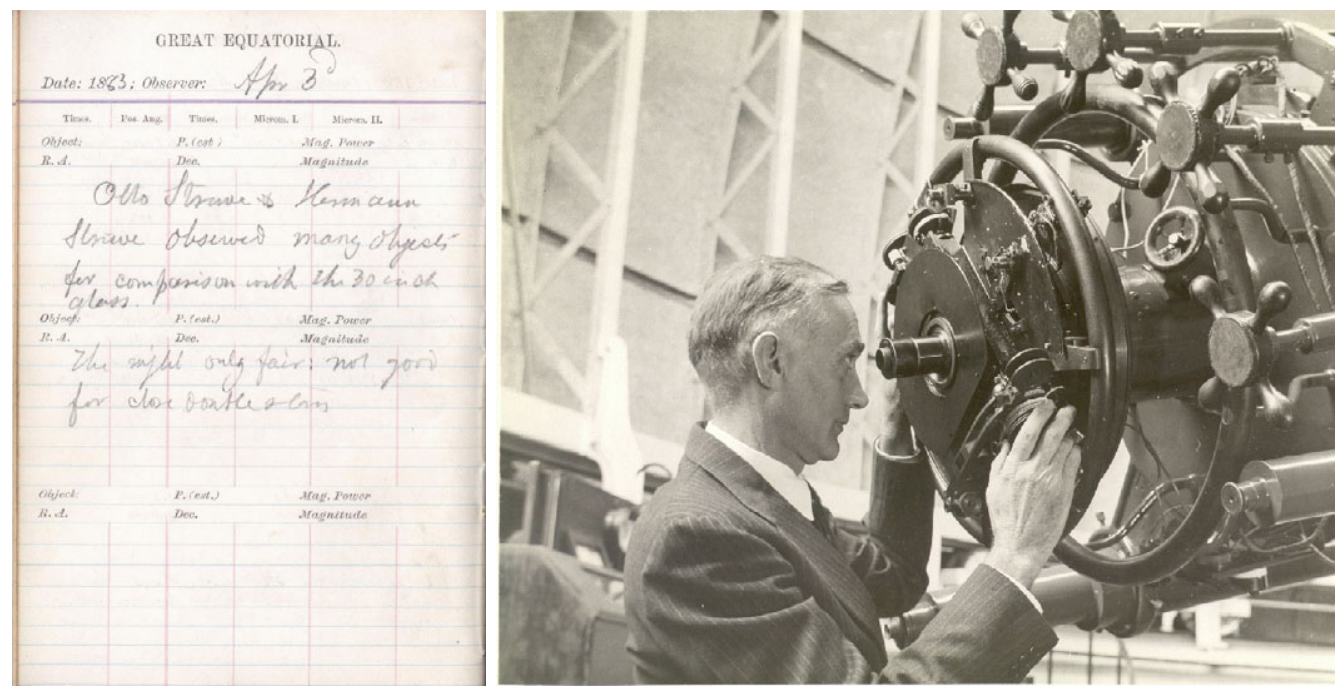

Figure 7. Left: Notes written by Otto and Herman Struve while testing the 26" (1883) in preparation for receiving the $30^{\prime \prime}$ made by Alvin Clark \& Sons. Right: U.S. Lyons with a micrometer on the $26^{\prime \prime}$.

\section{How do I get one?}

Copies of the US Naval Observatory Double Star CD, 2006.5 are available. Simply fill out the web form at http://ad.usno.navy.mil/wds/cd_request.html.

\section{Acknowledgements}

B.D.M. is supported by NASA under JPL task order \# NMO710776. W.I.H. is supported by the Office of Naval Research, Global by grant N00014-06-1-1054. We gratefully acknowledge this support.

\section{References}

Aitken, R.G. 1932, New General Catalogue of Double Stars within 121 degrees of the North Pole, Carnegie Institution of Washington

Burnham, S.W. 1906, General Catalogue of Double Stars within 121 degrees of the North Pole, Carnegie Institution of Washington

Finsen, W.S. 1934 Union Obs. Circular 4, 23

Finsen, W.S. 1938 Union Obs. Circular 4, 66

Finsen, W.S. \& Worley, C.E. 1970 Republic Obs. Circular 7, 203

Hartkopf, W.I., Mason, B.D., \& Worley, C.E., 2001, AJ 122, 3472

Jeffers, H.M., van den Bos, W.H., \& Greeby, F.M. 1963, Index Catalogue of Visual Double Stars, 1961.0, Pub. of the Lick Observatory, 21

Mason, B.D., Wycoff, G.L., Hartkopf, W.I., Douglass, G.G., \& Worley, C.E. 2001, AJ 122, 3466

Worley, C.E. 1963 Pub. U.S. Naval Obs. 18, pt. 3

Worley, C.E., \& Douglass, G.G. 1984, Washington Visual Double Star Catalog, 1984.0, U.S. Naval Observatory, Washington

Worley, C.E., \& Douglass, G.G. 1997, A\&AS 125, 523

Worley, C.E. \& Heintz, W.D. 1983 Pub. U.S. Naval Obs. 24, pt. 7

Worley, C.E., Mason, B.D., \& Wycoff, G.L., 2001, AJ 122, 3482 